\title{
Case of a floating right atrial thrombus successfully treated with fibrinolysis
}

\author{
Chloé Bernard, ${ }^{1}$ Olivier Bouchot, ${ }^{1}$ Marjolaine Georges, ${ }^{2}$ Marie Catherine Morgant $^{1}$ \\ ${ }^{1}$ Department of Cardiac Surgery; ${ }^{2}$ Department of Pneumology, Dijon University Hospital, Dijon, France
}

\begin{abstract}
Mobile right atrial thrombi are at high risk of causing massive pulmonary embolism and are a medical emergency. This type of thrombus is identified in about $4-18 \%$ of pulmonary embolism cases. The presence of a free-floating embolic mass in right atrial or ventricle is often mortal: the death rate can reach $27 \%$. Although surgery is commonly indicated, fibrinolysis is a therapeutic alternative. Here, a 58-year-old man presented to the emergency department for acute dyspnea associated with a painful right leg. Initial exams showed a shunt effect on blood gases with increased brain natriuretic peptide. Transthoracic echography (TTE) found a free-floating thrombus in the right atrium with signs of pulmonary hypertension and right ventricular dysfunction. Bilateral pulmonary embolism was confirmed by computed tomography angiography. Because there were signs of acute cor pulmonale and no contraindications, treatment with systemic fibrinolysis was decided. The treatment was delivered in intensive care unit and a cardiac surgical team was available. Subsequent clinical improvement was observed. TTE follow up at 12 hours revealed complete thrombus dissolution. There were no complications, in particular no hemorrhage. The patient was discharged after eleven days. At 6 months of follow-up, outcomes with oral anticoagulation therapy were satisfactory. Scintigraphy found good symmetrical perfusion of both lungs with stackable ventilation. TTE at 1 year showed preserved left and right ventricular function with no sign of pulmonary arterial hypertension and no thrombus recurrence. Systemic fibrinolysis appears to be a good alternative to surgery in this case.
\end{abstract}

Correspondence: Marie Catherine Morgant, Department of Cardiac Surgery, Dijon University Hospital, 2 Boulevard du Maréchal de Lattre de Tassigny, 21000 Dijon, France.

E-mail: mariecatherine.morgant@chu-dijon.fr

Key words: Fibrinolysis; Right atrium thrombus; Free-floating thrombus; Pulmonary embolism.

Contributions: the authors contributed equally.

Conflict of interest: the authors declare no potential conflict of interest.

Funding: none.

Received for publication: 7 September 2018.

Revision received: 13 February 2018.

Accepted for publication: 3 January 2019.

This work is licensed under a Creative Commons Attribution 4.0 License (by-nc 4.0).

(C) Copyright C. Bernard et al., 2019

Licensee PAGEPress, Italy

Emergency Care Journal 2019; 15:7813

doi:10.4081/ecj.2019.7813

\section{Introduction}

Deep vein thrombosis and pulmonary embolism (PE) are common, but right heart thrombus (RHT) is detected in less than $5 \%$ of cases of thromboembolic disease. ${ }^{1-2} \mathrm{PE}$ with RHT is associated with poor outcomes, especially in the absence of immediate treatment. ${ }^{1-4}$ Indeed, the majority of deaths occur in the first 24 hours (62\%). However, optimal management of PE with RHT remains unclear and there is a lack of randomized studies to support recommendations for disease management. ${ }^{2}$

\section{Case Report}

A 58-year-old Caucasian man presented with a one-week history of progressively worsening dyspnea and orthopnea (NYHA IV), with right lower limb pain. The patient's history revealed high blood pressure, dyslipidemia and sleep apnea, but no history of atrial fibrillation or thromboembolic disease (deep vein thrombosis or PE). He was not taking an anticoagulant or antiplatelet treatment. At the emergency room, patient had tachycardia (110 beats/min), tachypnea (35/min) and oxygen saturation was $88 \%$ while the patient was breathing ambient air. Arterial blood pressure was $102 / 55 \mathrm{mmHg}$. Electrocardiogram showed sinus tachycardia and S1Q3 waves. Blood gas analysis revealed hypoxemia $\left(\mathrm{PaO}_{2}\right.$ : $67.2 \mathrm{mmHg}$ ) and hypocapnia $\left(\mathrm{PCO}_{2:} 30.1 \mathrm{mmHg}\right)$ as a result of the shunt effect. The laboratory test results showed: i) Creatinine $=$ $177 \mu \mathrm{mol} / \mathrm{L}$ and urea $=9.2 \mathrm{mmol} / \mathrm{L}$, glomerular filtration rate $=37$ $\mathrm{ml} / \mathrm{min} / 1.73 \mathrm{~m}^{2}$; ii) NT-proBNP $=4978 \mathrm{ng} / \mathrm{L}$ and troponin $=0.18$ $\mu \mathrm{g} / \mathrm{L}$ (reference limit $<0.10$ ); iii) D-dimer : 20,000 $\mu \mathrm{g} / \mathrm{L}, \mathrm{C}$-reactive protein: 28 ; iv) Prothrombin ratio: $75 \%$ and activated thromboplastin time: 1.68 .

The patient was transferred to intensive care in the pneumology unit with a strong suspicion of pulmonary embolism. Computed tomography (CT) angiography confirmed the diagnosis of massive PE with proximal obstruction of both pulmonary arteries and revealed a right atrial thrombus (Figure 1). Transthoracic echocardiography (TTE) showed a free-floating serpentine thrombus within the right atrium (Figure 2) measuring $5 \mathrm{~cm} \times 3 \mathrm{~cm}(1.97$ " $\times 1.18$ ") and protruding through the tricuspid valve into the right ventricle during diastole. There was right ventricular dysfunction with high filling pressure and signs of acute cor pulmonale (paradoxical septal motion, pulmonary hypertension).

There was no contraindication for systemic fibrinolysis, so a multidisciplinary team decided to initiate systemic fibrinolysis without delay. The patient was treated with Alteplase $(10 \mathrm{mg}$ in 2 min then $90 \mathrm{mg}$ in $110 \mathrm{~min}$ ) in an accelerated regimen administered over $2 \mathrm{~h}$, as recommended by European guidelines. ${ }^{2}$ Intravenous heparin with electrical syringue (25200 UI/24hours) was given before, during and after administration of Actilyse.

During the procedure, the patient was monitored in the cardiac intensive care unit. He remained hemodynamically stable and respiratory capacities quickly improved. There was no hemorrhagic complication. 
Control TTE at 12 hours revealed complete dissolution of the right atrial thrombus (Figure 3) but persistent signs of acute cor pulmonale (moderate tricuspid regurgitation, right ventricular (RV) dilation, pulmonary hypertension, and paradoxical septal motion with preserved right ventricular function).

TTE at discharge (10 days) highlighted improved pulmonary hypertension (pulmonary artery pressure $36 \mathrm{mmHg}$ ), moderate tricuspid regurgitation and a decrease in right atrial dilation.

After 3 months, only the right atrial dilation remained.

Ventilation/perfusion scintigraphy performed at 6 months showed symmetrical perfusion of the two lungs without ventilation mismatch.

TTE at 1 year showed a preserved left and right ventricular function without dilation or hypertrophy. Only a mild apical septal hypokinesia persisted. There was no sign of pulmonary arterial hypertension, no atrial dilation, no tricuspid insufficiency and no thrombus recurrence.

The etiologic investigation included a thrombophilia screening (which showed an increased level of homocysteine), a body scanner which did not find neoplasia and a venous Doppler exam which revealed deep vein thrombosis in the right leg.

\section{Discussion}

The European guidelines for the management of pulmonary embolism are clear. In case of PE with hemodynamic instability (arterial hypotension, RV dysfunction) and no contraindication, systemic fibrinolysis is the first-line treatment. ${ }^{3}$ However, because there are few cases reported in the literature (some case reports or retrospective cohorts but no randomized or prospective studies), the management of RHT is still matter of debate. Even the European register of RHT included a limited number of patients because of the low incidence of PE with RHT. ${ }^{2}$ No study has provided sufficient level of evidence to provide specific recommendations on the proper management of these patients.

In the literature, thrombolysis with intravenous heparin is the first suggested treatment for RHT regardless of the hemodynamic stability. ${ }^{4}$ The advantage of this treatment is the lysis of floating thrombus and the associated treatment of deep vein thrombosis 3,5 and it is also less invasive than surgery.

In case of contraindications, such as recent surgery, intravenous heparin alone can be suggested as an effective therapeutic alternative.

Early surgical embolectomy continues to be useful when thrombus is associated with a patent foramen oval seeing as fibrinolysis poses a risk of systemic embolization of a partially fragmented clot. ${ }^{5,6}$

There are therefore advantages and disadvantages to each treatment: systemic fibrinolysis and its bleeding risks, surgical embolectomy and its perioperative risks and isolated curative anticoagulation and its longer duration of action. From the data available in the literature, systemic fibrinolysis seems to be an effective and safe treatment for the management of PE with RHT and hemodynamic instability. However, no existing study has been able to demonstrate the superiority of fibrinolysis or surgery in unstable hemodynamic patients. Therefore, treatment of these patients should be decided by a multidisciplinary team, taking into account individual operative and bleeding risks and the accessibility of the procedure (availability of a cardiac surgery unit).

Consensus does exist on the need for urgent treatment, especially in patients with hemodynamic instability. Indeed, Koć et al. ${ }^{6}$ reported that the majority of deaths $(62 \%)$ occurred within the first $24 \mathrm{~h}$ and that hemodynamic instability is strongly associated with an increased risk of death $(\mathrm{P}<0.001)$.

This case report also highlights the value of TTE in the management of patients with massive PE. The majority of deaths occur within the first $24 \mathrm{~h}$, and any delay in the diagnosis and treatment of RHT could thus have significant repercussions on morbidity and mortality. TTE has the advantage over the CT angiography to diagnose the thrombus but also to evaluate its impact on the right heart. The incidence of diagnosed RHT is low (about $4 \%$ of PE) but not insignificant in view of PE incidence. It is possible that without TTE small thrombi (with possibly less hemodynamic impact) go undiagnosed and therefore untreated, increasing the risk of death, chronic cor pulmonale, and, in patients with patent foramen ovale, systemic embolization (Appendix).

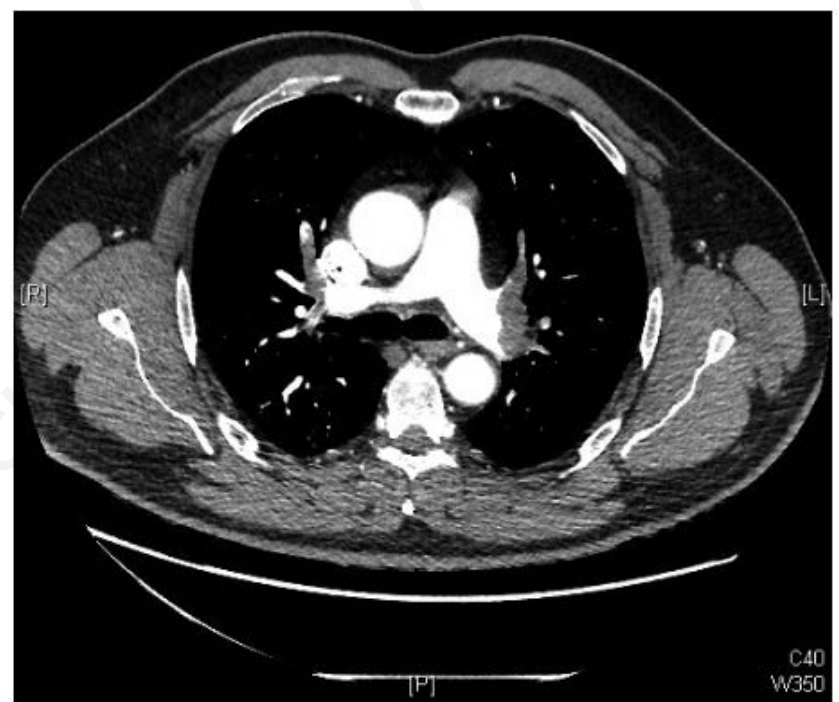

A

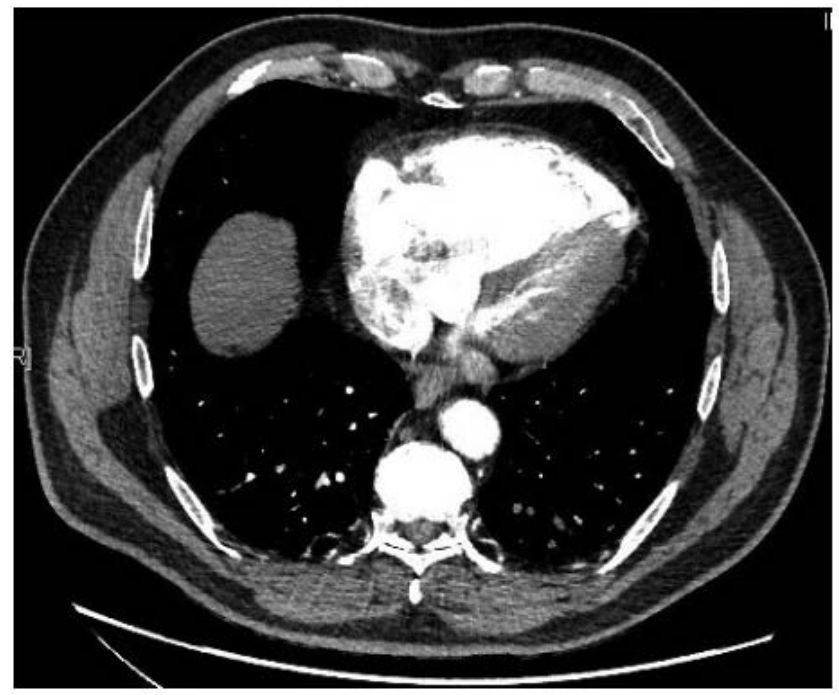

B

Figure 1. Injected tomodensitometry, in axial view. Massive pulmonary embolism with proximal obstruction of the both pulmonary arteries (A) and revealed a right atrium thrombus (B). 


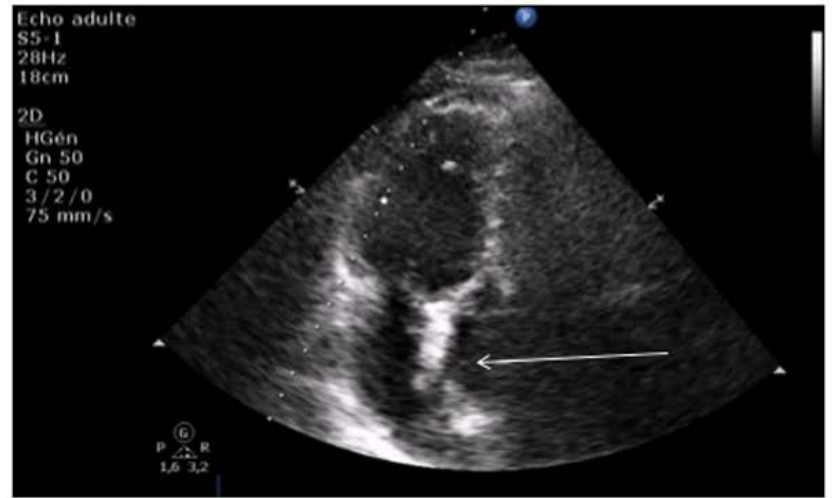

A

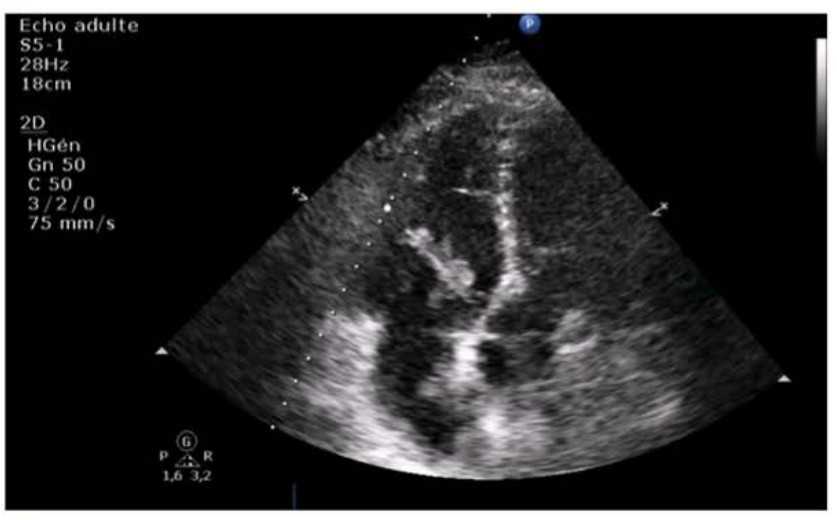

B

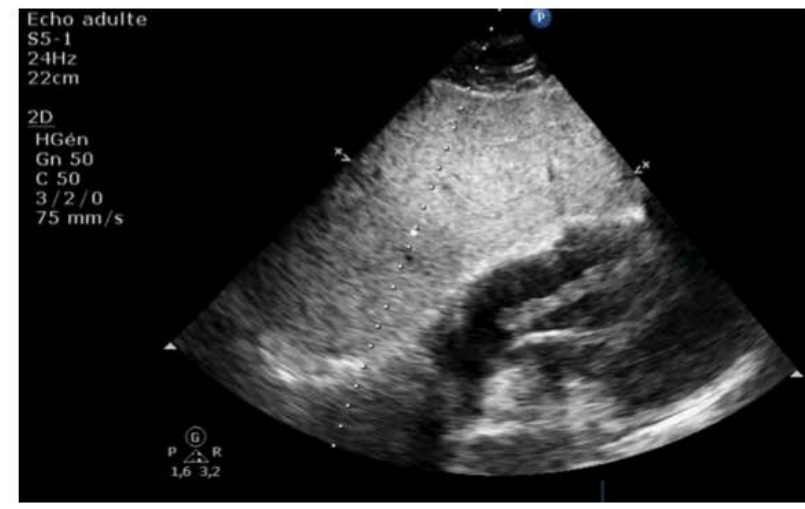

C

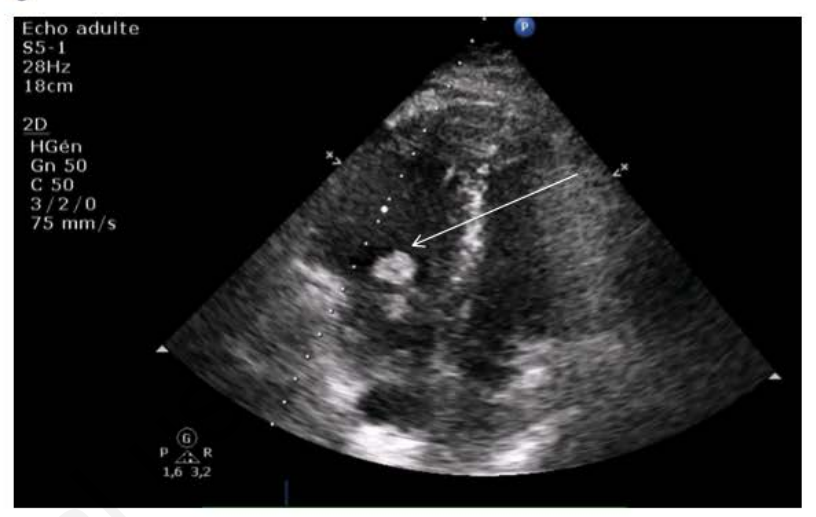

D

Figure 2. Transthoracic echography: four cavities and long axes cross section. (A) Free floating thrombus in the atrium protruding into the right ventricle during diastole through tricuspid valve; (B-D) different views.

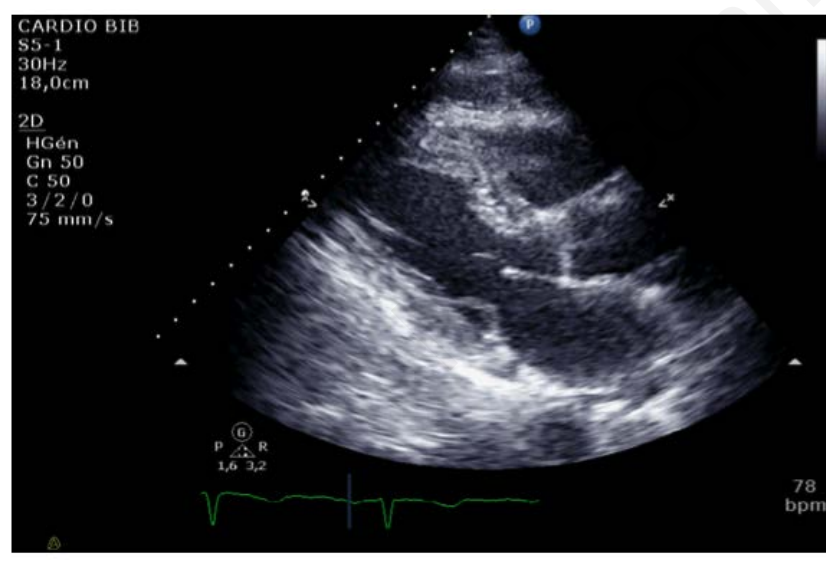

Figure 3. Control transthoracic echocardiography at 12 hours: complete right atrium thrombus dissolution.

\section{Conclusions}

Systemic fibrinolysis appears to be a safe and effective treatment for the management of PE with RHT and hemodynamic instability. Surgical embolectomy continues to have a role in the management of these patients, especially in case of contraindication or failure of fibrinolysis. TTE is essential for the diagnosis of
RHT in patients with massive pulmonary embolism, and gives clinicians the possibility to evaluate impact on the right ventricle and to monitor patients appropriately over the course of treatment.

\section{References}

1. Bodian $\mathrm{M}, \mathrm{Ba}$ FG, Jobe $\mathrm{M}$, et al. Fatal evolution of a huge right atrial free-floating thrombus. Clin Case Rep 2013;1:63-5.

2. Benjamin MM, Afzal A, Chamogeorgakis T, Feghali GA. Right atrial thrombus and its causes, complications, and therapy. Proc (Bayl Univ Med Cent) 2017;30:54-6.

3. Dzudovic B, Obradovic S, Rusovic S, et al. Therapeutic approach in patients with a floating thrombus in the right heart. J Emerg Med 2013;44:e199-205.

4. Torbicki A, Galié N, Covezzoli A, et al. Right heart thrombi in pulmonary embolism: results from the International Cooperative Pulmonary Embolism Registry. J Am Coll Cardiol 2003;41:2245-51.

5. De Freitas S, Connolly C. Right atrial thrombus and massive pulmonary embolism refractory to thrombolytic therapy: A case report. Int J Surg Case Rep 2016;25:212-4.

6. Koć M, Kostrubiec M, Elikowski W, et al. Outcome of patients with right heart thrombi: the Right Heart Thrombi European Registry. Eur Respir J 2016;47:869-75. 